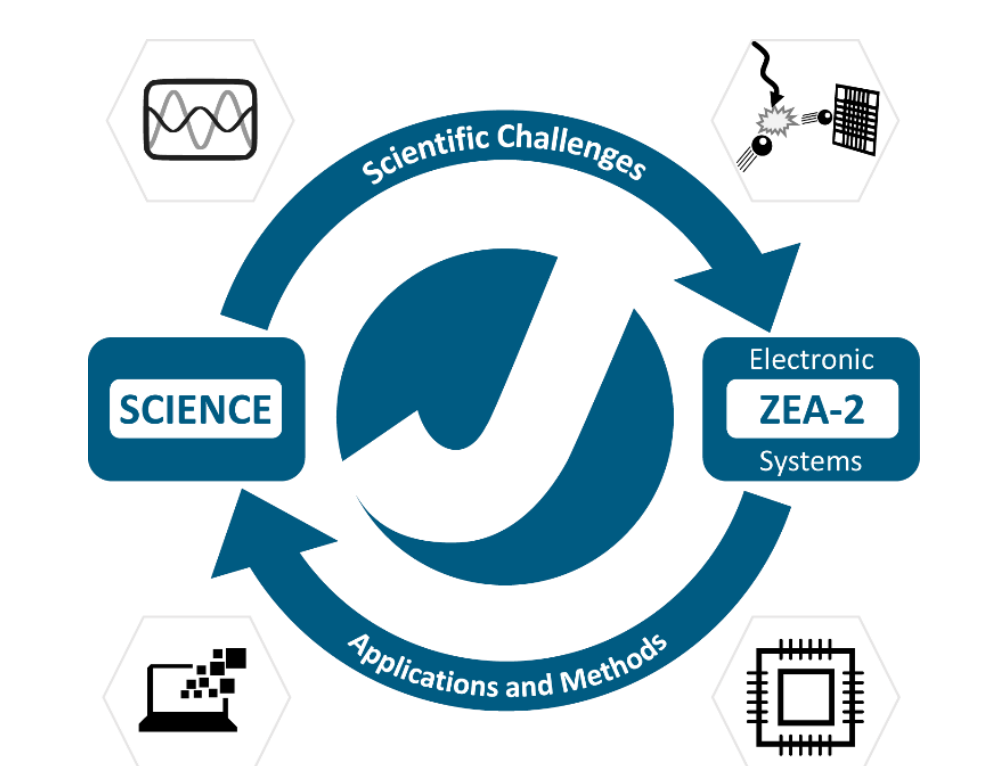

\title{
Smooth wavelength transition in a Lorentz force based actuator system for turbulence research
}

\author{
S. Völkel ${ }^{1}$, R. Heil ${ }^{1}$, M. Schiek ${ }^{1}$, M. Schlösser ${ }^{1}$, W. Silex ${ }^{1}$, S. van Waasen ${ }^{1,2}$
}

1. Central Institute for Electronic Systems (ZEA-2), Forschungszentrum Jülich GmbH, Jülich, Germany

2. University of Duisburg-Essen, Faculty of Engineering, Duisburg, Germany

\section{Introduction}

For the development of robust methods to significantly reduce friction drag caused by the turbulent boundary layer the DFG research group FOR1779 analyses the effect of transversal waves on an extended aluminum surface in wind tunnel experiments.

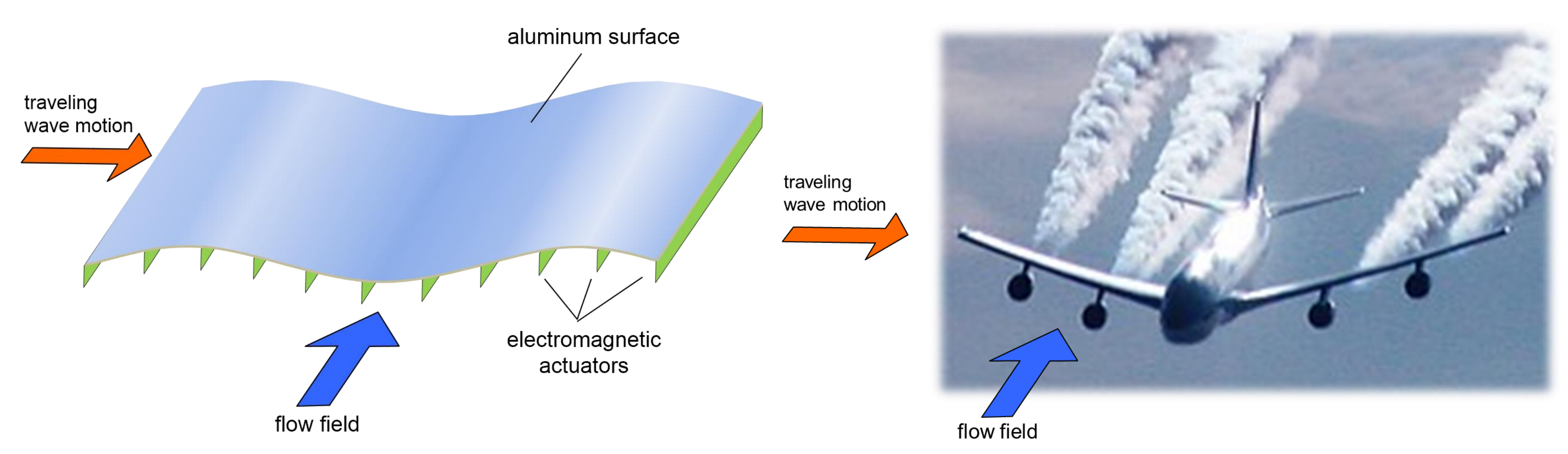

The transversal surface waves running in the span direction (orange arrow) cause an interaction with the near-surface flow field (blue arrow).

An actuation system developed by ZEA-2 generating the transversal waves by phase-shifted driven, parallel orientated and uniformly distributed electromagnetic actuators. These actuators are glued to the aluminum surface and hence are mechanically coupled.

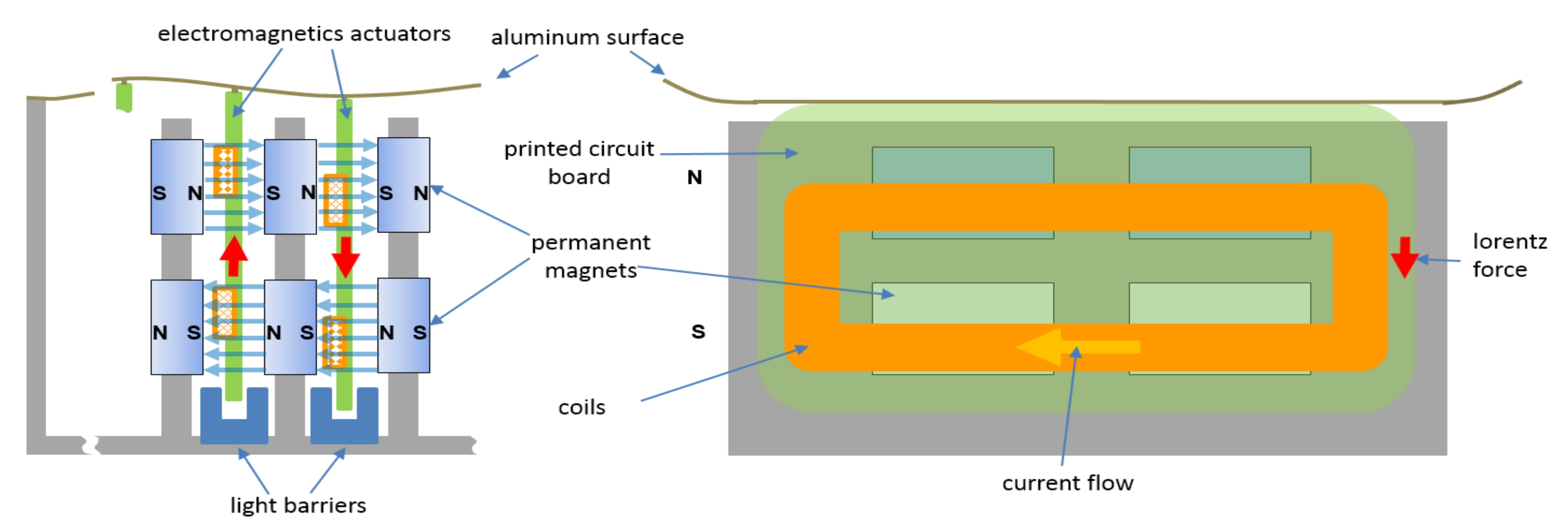

Sketch of the electromagnetic actuator system.

For the generation of the running transversal waves, the actuators are driven by the FPGA RIO board PXI-7831 R from National Instruments via an analogue amplifier front-end. The Virtex-II FPGA Chip included in this board provides automated calculated smooth signal transition when adapting the signal characteristics on the external change of the wave parameters amplitude, frequency and wavelength. This prevents discontinuous signals slope with high frequency interference on the aluminum surface.

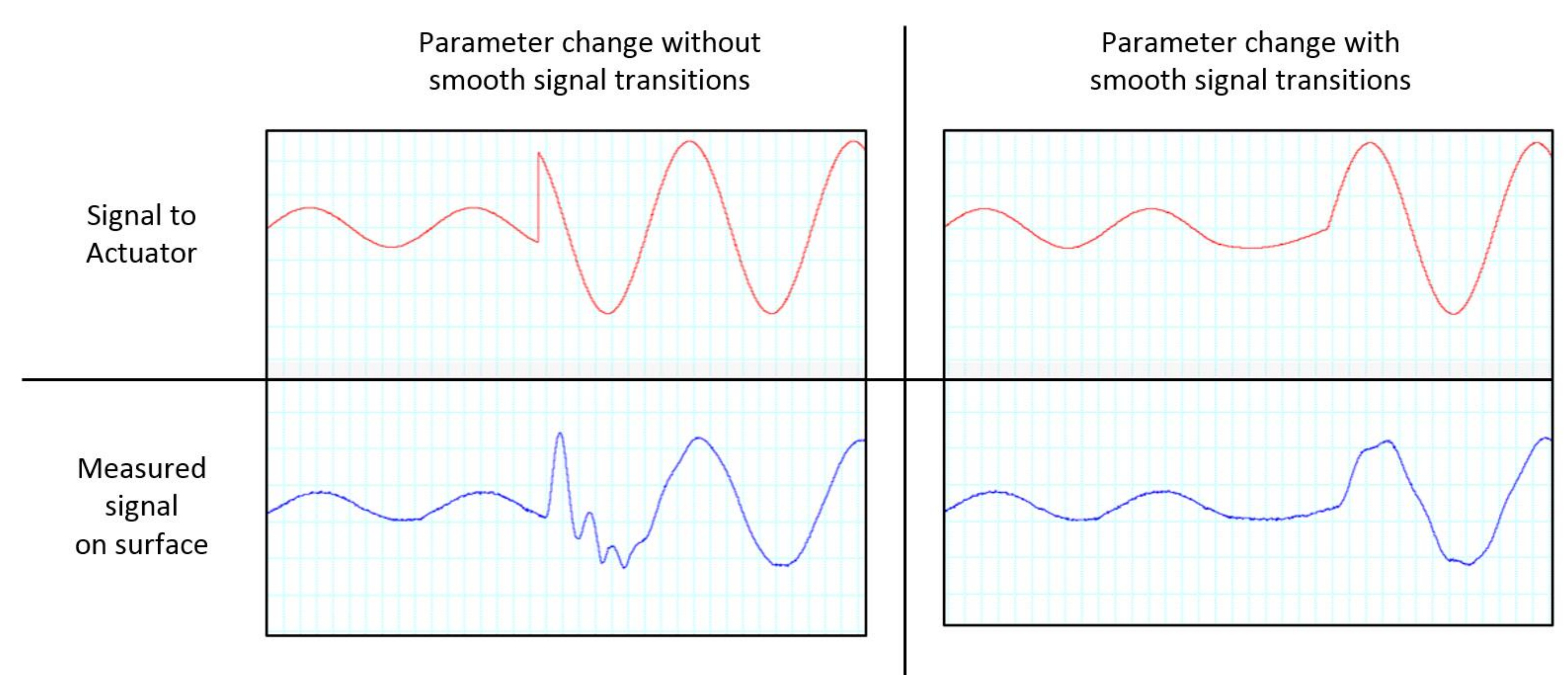

The high frequency interference by rapid parameter changes without smooth signal transition is shown on the left versus a parameter change with calculated smooth signal transitions on the right.

We implemented the smooth wavelength transition based on direct digital synthesis (DDS). This method ensures a smooth wavelength transition by means of an intelligent manipulation of the readout steps of a stored waveform look-up table.

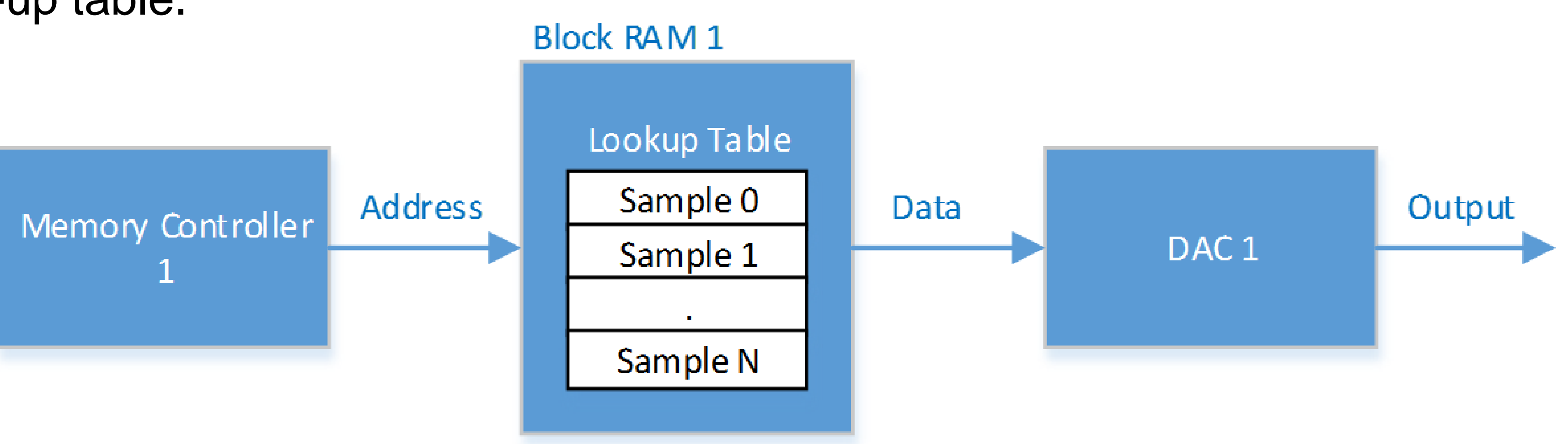

\section{Generation of Transversal Waves}

Instead of continuously calculating the phase-shifted signal sequences generating the traveling waves, one sinusoidal wave period is stored once in the look-up table at the block RAM of the FPGA: The phase shifted signals are generated by a basic function of the DDS. In this function, integrated memory controllers run through the look-up tables with calculated steps CS $_{\text {MEM }}$ depending on the desired output frequency $f$, the variable loop clock of the memory clk and the size of the look-up table $N$.

$$
C S_{M E M}=f \times c l k \times N
$$

By assuming that the stored values in the look-up table can be mapped to the unit circuit, each data point has its own phase information that can be evaluated. This means that by setting different starting addresses of the memory controllers with the same clock, a phase shift between the signals can be generated.
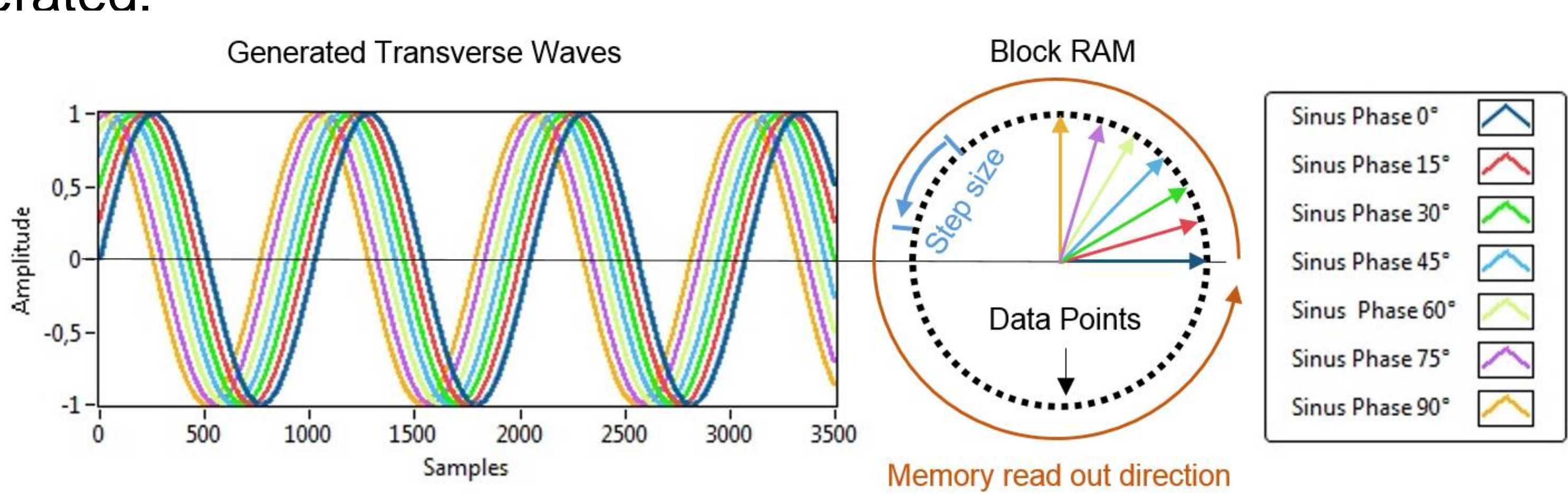

The generated transverse waves over phase shift sine signals emerge by setting different starting addresses of the memory controllers.

\section{Smooth Wavelength Transition Method}

As mentioned in the previous chapter, the look-up table is sampled with different step sizes as a function of the wave frequency. Each amplitude value of the signal has its own sample in the memory which is known as the memory point $\mathrm{P}_{0}$ combining an amplitude value to the sample at $\mathrm{S}_{0}$,

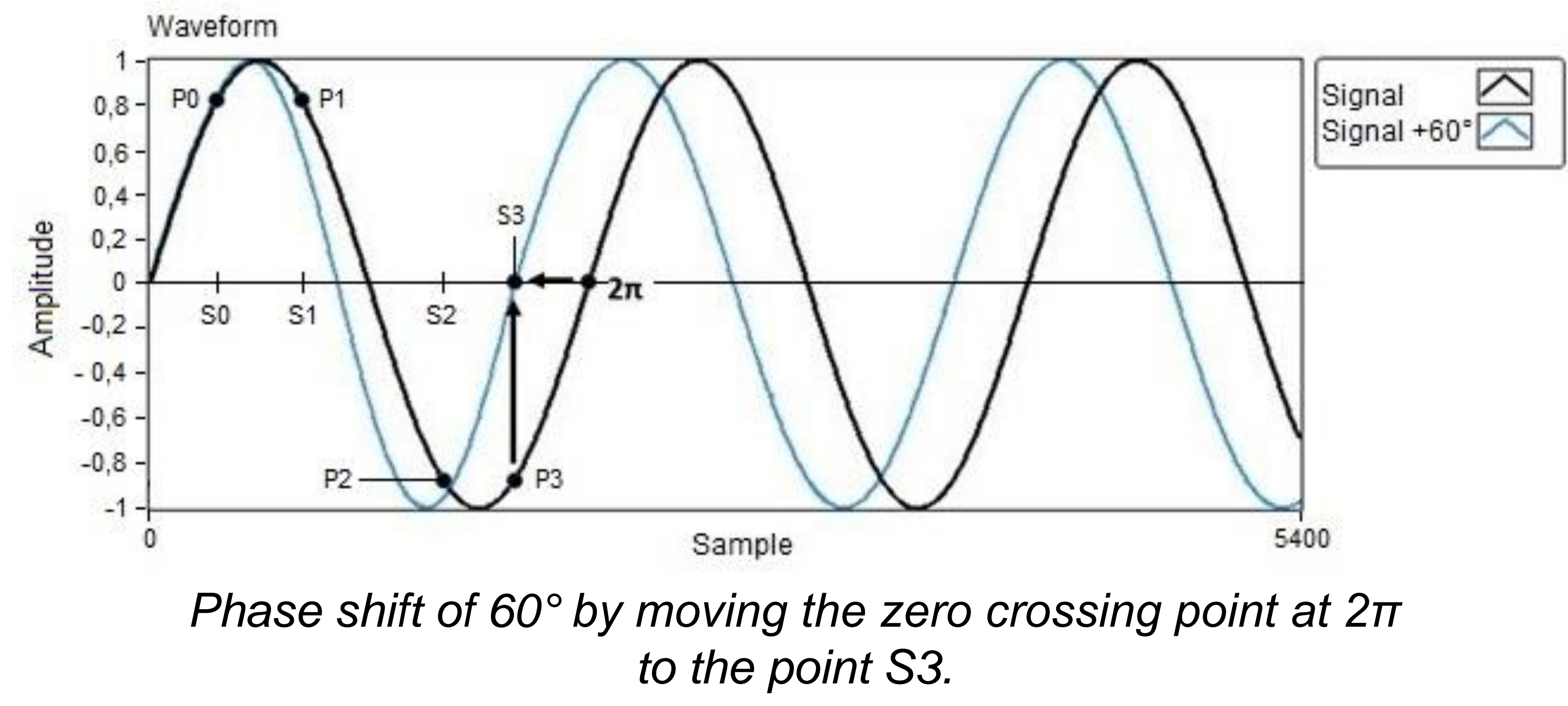

The method of changing the phase, without high-frequency interferences caused by rapid changes in the voltage and its effects on the aluminum surface, is a negative or positive time shift of the signal by moving the zero crossing point by increasing or decreasing the readout steps while the memory controller is at the sample of the amplitude value $P_{n}$ of the changing phase.

$$
P_{n}=\sin \left(2 \times \pi \times f+(\pi \times \text { phase }) /\left(180^{\circ}\right)\right)
$$

This assumption could be made by the characteristic of a periodic and zerocentered signal that the phase value always appears positive or negative every quartered period. By the time the calculated phase value reaches the memory point $S_{0}$, the zero crossing following after a half-period is shifted to the sample position of $\mathrm{S}_{3}$. This results in a faster sampling $\mathrm{CS}_{\mathrm{New}}$ of the memory so that the zero crossing is reached earlier.

$$
C S_{N e w}=\left(\left(N-S_{0}\right) \times C S_{M E M}\right) / N
$$

\section{Conclusion}

The developed electromagnetic actuator system is used for experiments to develop robust methods for turbulent friction resistance reduction by transversal surface waves. In case of changing inflow conditions waveform parameters like wavelength have to be adapted on the fly. For this, the driving system of the electromagnetics actuators uses automatically calculated smooth transients between various surface waves which allows an efficient operation of the necessary parameter studies in the wind tunnel. The smooth transient avoids the occurrence of high-frequency disturbances into the flow field and thus eases the application of special control algorithms ensuring the precision of the waveform (see Poster by Seidler et al., B-12-287). 